\title{
Innovative 3D Animations for Teaching Electromagnetic Field Theory and its Mathematics in Undergraduate Engineering
}

\author{
Hennig, Markus ${ }^{\mathrm{a}}$ and Mertsching, Bärbel ${ }^{\mathrm{a}}$
}

GET Lab, University of Paderborn, Germany

\begin{abstract}
In this work, an innovative approach for the design and structuring of teaching videos systematically using $3 D$ animations is presented. The approach focuses on the quantitative description of electromagnetic fields and the mathematical methods and competencies required for this purpose, exemplarily with regard to an undergraduate electrical engineering course during the initial phase of corresponding degree programs. An essential part of this course is the spatial and time-dependent description of electromagnetic fields. For this purpose, students have to work with multiple integrals in 3D space and in different coordinate systems. Such subjects are typically covered only later in mathematics courses and without a technical context, therefore leading to major difficulties for many students. The videos presented in this work are intended to support students and lecturers to work with these subjects in an instructive fashion. The $3 D$ animations allow for effectively clarifying complex connections between technical and mathematical aspects. The videos and their specific design are discussed with regard to didactic and technical considerations. Additionally, their integration with existing interventions for the course is described.
\end{abstract}

Keywords: 3D Animations; Electromagnetic Fields; Electrical Engineering, Engineering Mathematics, E-learning. 


\section{Introduction}

The quantitative description of electromagnetic fields usually requires students to work with multiple integrals in different coordinate systems. The application of corresponding integral equations is connected with comprehensive spatial reasoning in 3D space. The resulting complexity of such subjects leads to major difficulties for many students (Hennig, Mertsching, \& Hilkenmeier, 2015). This is a particular issue when electromagnetic fields are covered in the initial phase of undergraduate engineering degree programs, where corresponding advanced mathematical subjects are typically addressed only later in mathematics courses. Electrical engineering degree programs cover electromagnetic fields in the initial phase because of their essential relevance for exhaustively understanding and describing most electrical phenomena, for example in terms of direct current networks. Additional challenges include restricted and heterogeneous mathematical expertise of incoming students, low self-directed learning readiness, and a different view on mathematics in comparison to school (Alpers et al., 2013).

Common approaches to respond to these challenges are the establishment of bridging courses, mathematics support centers, and restructuring the curriculum (Biza et al., 2016). While bridging courses are usually focusing on basic mathematical subjects, mathematics support centers are an additional and uncoupled intervention so that they are possibly not attractive. Restructuring the curriculum competes with the systematic development of theory in mathematics and engineering courses, respectively. In this work, video instruction systematically using 3D animations is presented as a partial solution to address above mentioned challenges. The videos are integrated with existing interventions for an undergraduate electrical engineering course, therefore not appearing as an uncoupled intervention. Additionally, time and place-independent learning are facilitated.

In comparison to the 2D case, 3D animations and visualizations allow for clarifying problem statements in 3D space "as they are". Reduced simplifications, such as 2D cross sections, are avoided. For example, if the 3D symmetry properties of a 3D technical setup have to be analyzed for mathematical modeling purposes, a 2D simplification might complicate this process. 3D studio animations as presented in this work can also be used to visualize technical models of electromagnetic fields, for example field lines of electric or magnetic flux, together with mathematical modeling aspects. In reality, such properties cannot be visually observed directly but only by indirect effects, such as forces between charged objects. This might lead to difficulties for students to build dynamic mental 3D models of electromagnetic fields. When the videos are integrated with the course, corresponding mathematics can be presented directly in the technical context and with a consistent mathematical notation. This is in contrast to mathematics courses, which are often taught "as a service" by mathematics departments to students from various fields, therefore changing notations and the lack of technical contexts might be an issue. 


\section{Didactic Classification}

The wide-spread interest in Massive Open Online Courses (MOOCs) and flipped-classroom approaches in recent years motivated further analysis of different production styles of video instruction in the literature. In (Guo, Kim, \& Rubin, 2014), the authors distinguish between six different video production styles, of which the so-called Khan-style (freehand writing on a digital drawing board with audio commentary, as extensively used by Khan Academy) and video capturing of class teaching are probably most common. To the best of the knowledge of the authors of this work, the specific production style and structuring of the videos presented in this work (systematic usage of 3D studio animations covering complex connections between technical and mathematical aspects) are not described in the literature. This is why the videos presented in this work are referred to as being innovative.

In general, it can be stated that multimedia animations and presentations can be an effective instrument to enhance the learning process. Nonetheless, it remains unclear under which principal conditions (such as specific learning goals) different production styles of teaching videos are most effective (Clark \& Mayer, 2016). For example, it has not been investigated in which cases 2D animations are more effective than comprehensive 3D animations. On the contrary, 3D animations and visualizations are used in science and engineering education since many years (Gilbert, 2005; McGrath \& Brown, 2005). Particularly representative in this context is the usage of $3 \mathrm{D}$ anatomy models in medical education. These are intended to support building dynamic mental 3D models of anatomical structures (Azer \& Azer, 2016). Similarly, 3D models of complex molecular structures are used in chemistry education, while 3D visualizations of geologic structures are used in geology education (Gilbert, 2005). Considering electromagnetic field theory, Dori and Belcher (2005) developed a broad range of 3D visualizations (also with a focus on student activation) for an introductory electromagnetism course. While the authors conclude that $3 \mathrm{D}$ visualizations can enhance the conceptual understanding of the course contents, they are not focusing on complex connections between technical and mathematical aspects.

Niss and Højgaard (2011) elaborated a set of eight characteristic (but mutually connected) mathematical competencies which can be necessary to work with mathematical problems. The videos presented in this work are explicitly focusing on three of these competencies in relation to the technical course contents (situated). To be more precise, the competency to handle mathematical symbols and formalisms, mathematical modelling competency, and the competency of mathematical reasoning are addressed. Referring to this, the competency to handle mathematical symbols and formalisms comprises the ability to decode and interpret symbolic mathematical language. For example, this competency is necessary to correctly choose the specific geometric object, such as an open or closed contour or surface, over which different multiple integrals have to be evaluated. The mathematical modelling competency involves the ability to translate (mathematize) objects and relations to a 
mathematical form as well as to work with the resulting models. For example, mathematical descriptions of infinitesimal elements of specific geometric objects have to be built to be used as differentials within different multiple integrals. In order to evaluate the resulting multiple integrals, it is necessary to be able to reason mathematically. For example, if the variables of a function to be integrated do not depend on the integration variables, these can be written in front of the integral so that the integration is simplified.

For the design and structuring of the videos presented in this work, among other considerations, the evidence-based principles of multimedia learning (Clark \& Mayer, 2016) are taken as instructional guidelines. The principles are based on the cognitive theory of multimedia learning which models - broadly formulated - information processing and integration when learning with multimedia instruction. In this theory, a multimedia presentation consists of words which can be written or spoken, and pictures which can be static or dynamic. The material presented is then processed by a separate visual and verbal channel, each with limited capacity. Finally, in order to achieve deep learning, active processing of information is necessary. This involves the selection, organization and mutual conversation of words and pictures. The application of the principles of multimedia learning derived from this theory is described in the next section.

\section{Video Design and Structuring}

The design and structuring of the videos presented in this work are exemplary described for a video about Gauss's law in electrostatics, see fig. 1 and fig. 2 in appendix for example images. The equation (right side in fig. 1a) states that the integration of the electric displacement field $\overrightarrow{\mathbf{D}}$ over a closed surface $A$ equals the therein enclosed free charge $Q$. In this example, $Q$ is given by a spherical volume charge with radius $R$ positioned in the origin of a Cartesian coordinate system. The video remains focused on this arrangement and does not include extraneous material (coherence principle). Explanations are given by audio comments and are not written on the screen again (modality/redundancy principle).

In order to apply the integral equation correctly, it is important to understand the concept of enclosed charge. This is why it is shown that a volume charge can be considered as an arrangement of many point charges (left side in fig. 1a). This leads to the conclusion that $\overrightarrow{\mathbf{D}}$ has to be calculated outside $(r>R)$ and inside $(r \leq R)$ the volume charge separately, where $r$ is the distance from the coordinate origin. The currently considered case is always specified at the top left to highlight the organization of the video (signaling principle). Next, a 3D symmetry analysis identifies the electric displacement field to be radially symmetrical and to only depend on $r$ (fig. 1b). It is therefore advantageous to describe the field in spherical coordinates to obtain a compact mathematical description. The spherical 
coordinate system is covered earlier and not included in this video (pre-training principle). The captions for the displacement field, the axis and other objects are positioned closed to their corresponding elements to point out their spatial contiguity (spatial contiguity principle). The animations are oftentimes segmented and stopped when giving long explanations or when explaining mathematical evaluation steps (segmenting principle).

Due to the spherical symmetry properties, it is advantageous (simplification of evaluation steps) to use a sphere as a closed surface. This process is illustrated in fig. 1c. It is now important to understand the correct formulation and the meaning of an infinitesimal area element in 3D space to be used as differential $\mathrm{d} \overrightarrow{\boldsymbol{A}}$. The verbal explanation and mathematical description (model) are presented simultaneously (temporal contiguity principle). The intervals of the integration variables (integration limits) have to be selected so that the spherical surface is completely closed. An animation illustrating this is shown in fig. 2 a. Note that such a process cannot be visualized in 2D. After explaining the evaluation of the integral (fig. 1b), the electric displacement field is calculated inside the volume charge. In comparison to the outside, the enclosed charge depends on the dynamic size of the closed surface. This concept is visualized by an animated shrinking of the surface. Finally, the resulting field description is presented (fig. 1c.).

The videos have a length of approx. 6 minutes (as suggested by Guo, Kim, \& Rubin, 2014). The audio comments are spoken by an experienced lecturer in conversational style based on a pre-written script (personalization/voice principle). It is intended to avoid the impression that the speaker himself does not understand the video contents (which might be the case for a professional speaker). The production of the videos is based on a script with draft sketches. For producing the videos, the open-source software Blender is used.

\section{Conclusion and Future Work}

The videos presented in this work allow for a comprehensive presentation of complex connections between technical and mathematical aspects in 3D space. Using traditional 2D animations and visualizations, such a dynamic presentation appears to be difficult.

The videos are integrated with an existing web-based learning platform. This platform is used within and outside the face-to-face courses to implement a blended learning scenario (for details see Hennig, Mertsching, \& Hilkenmeier, 2015). The connection with activating elements (cf. Loviscach, 2014) is planned. An important next step is the systematic evaluation with regard to the acceptance and effectiveness of the videos, especially because of the limited research available for such specific videos. 


\section{References}

Alpers, B. A., Demlova, M., Fant, C.-H., Gustafsson, T., Lawson, D., et al. (2013). A framework for mathematics curricula in engineering education. European Society for Engineering Education (SEFI). Retrieved from https://dspace.lboro.ac.uk/2134/14747.

Azer, S. A., \& Azer, S. (2016). 3D anatomy models and impact on learning: A review of the quality of the literature. Health Professions Education, 2(2), 80-98. doi:10.1016/ j.hpe.2016.05.002.

Biza, I., Giraldo, V., Hochmuth, R., Khakbaz, A., \& Rasmussen, C. (2016). Research on teaching and learning mathematics at the tertiary level (ICME-13 Topical Surveys). doi:10.1007/978-3-319-41814-8_1.

Clark, R. C., \& Mayer, R. E. (2016). E-learning and the science of instruction: Proven guidelines for consumers and designers of multimedia learning (4th ed.). Hoboken, New Jersey: John Wiley \& Sons.

Dori, Y. J., \& Belcher, J. (2005). Learning electromagnetism with visualizations and active learning. In J. K. Gilbert (Ed.), Visualization in science education (1st ed.) (pp. 187216). Dordrecht, Netherlands: Springer.

Gilbert, J. K. (Ed.). (2005). Visualization in science education (1st ed.). Dordrecht, Netherlands: Springer.

Guo, P. J., Kim, J., \& Rubin, R. (2014). How video production affects student engagement: an empirical study of MOOC videos. Proc. of the 1st ACM Conference on Learning, Atlanta, Georgia, USA. doi:10.1145/2556325.2566239.

Hennig, M., Mertsching, B., \& Hilkenmeier, F. (2015). Situated mathematics teaching within electrical engineering courses. European Journal of Engineering Education, 40(6), 683-701. doi:10.1080/03043797.2014.1001820.

Loviscach, J. (2014). Friendly handmade explanation videos. Proc. of the European MOOC Stakeholder Summit (EMOOCs), Lausanne, Switzerland.

McGrath, M. B., \& Brown, J. R. (2005). Visual learning for science and engineering. IEEE Computer Graphics and Applications, 25(5), 56-63. doi:10.1109/mcg.2005.117.

Niss, M., \& Højgaard, T. (Eds.). (2011). Competencies and mathematical learning. Ideas and inspiration for the development of mathematics teaching and learning in Denmark. (IMFUFA tekst nr. 485/2011). Roskilde: Roskilde University. 


\section{Appendix}

a)

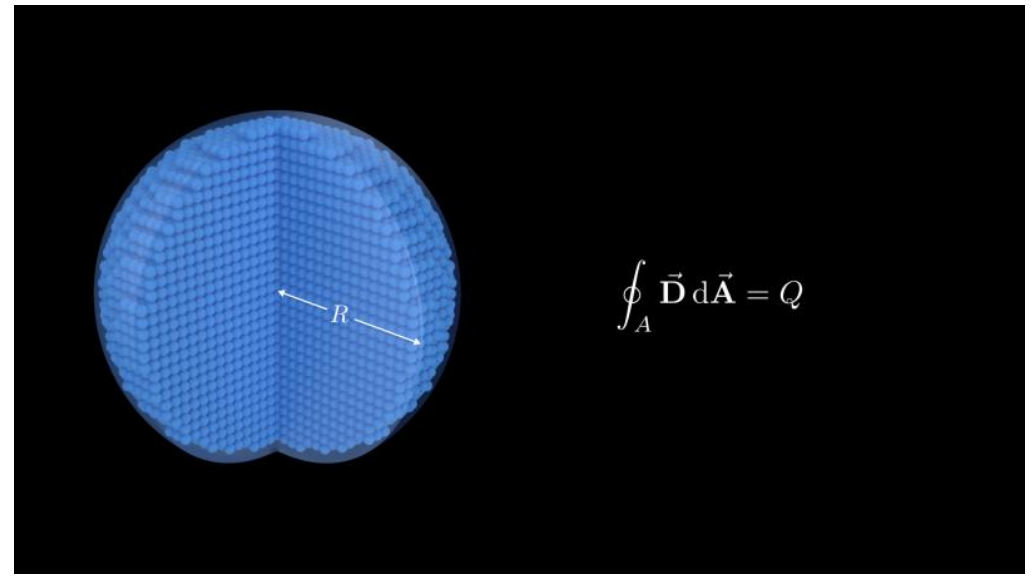

b)

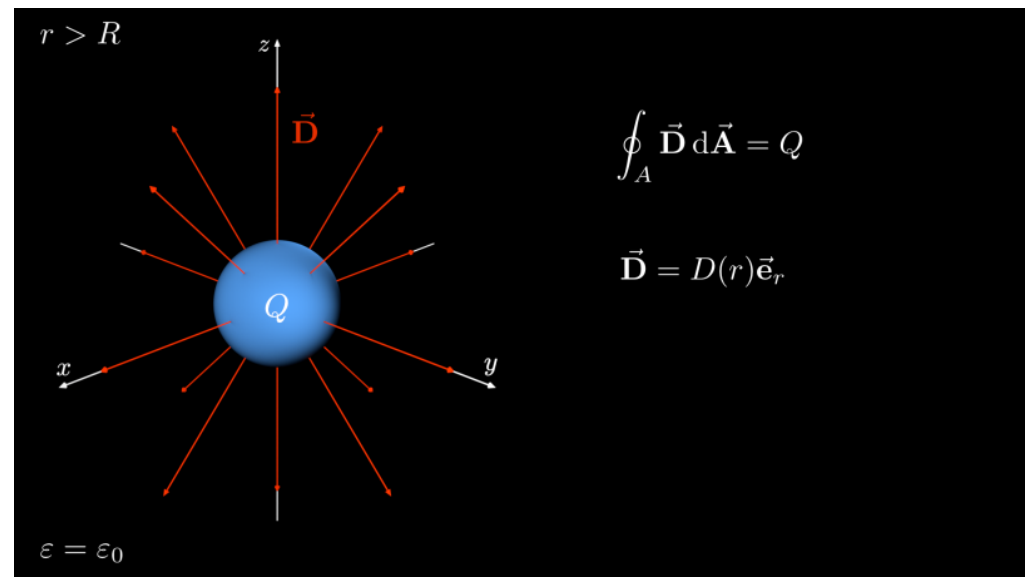

c)

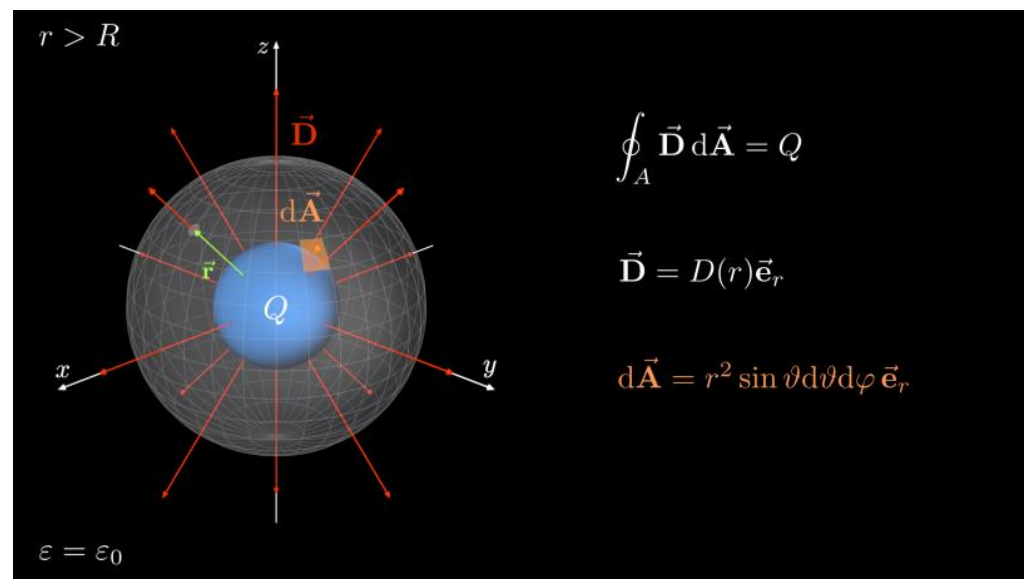

Figure 1: Selected frames from one of the teaching videos developed in this work, see text for details. 
a)

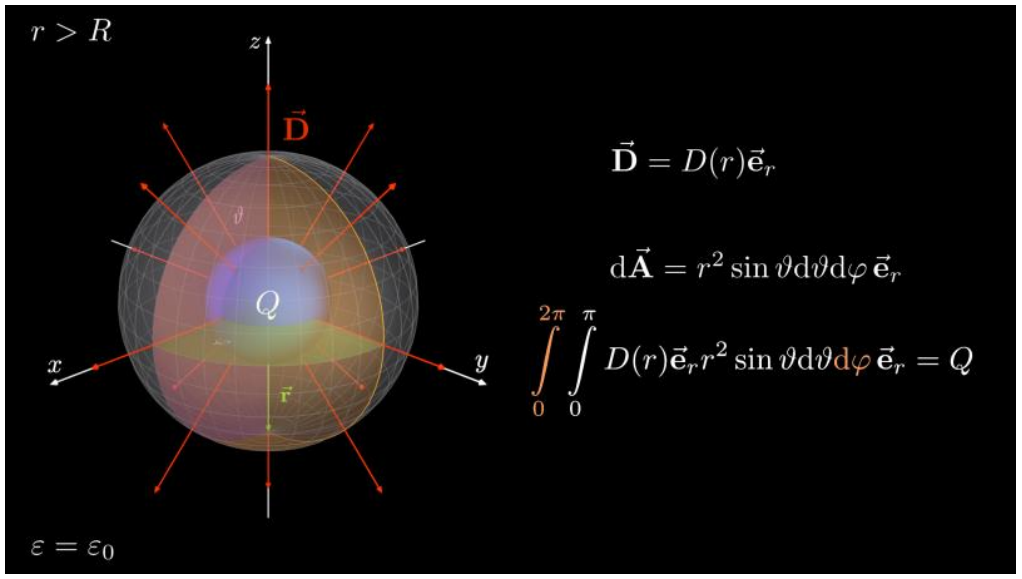

b)

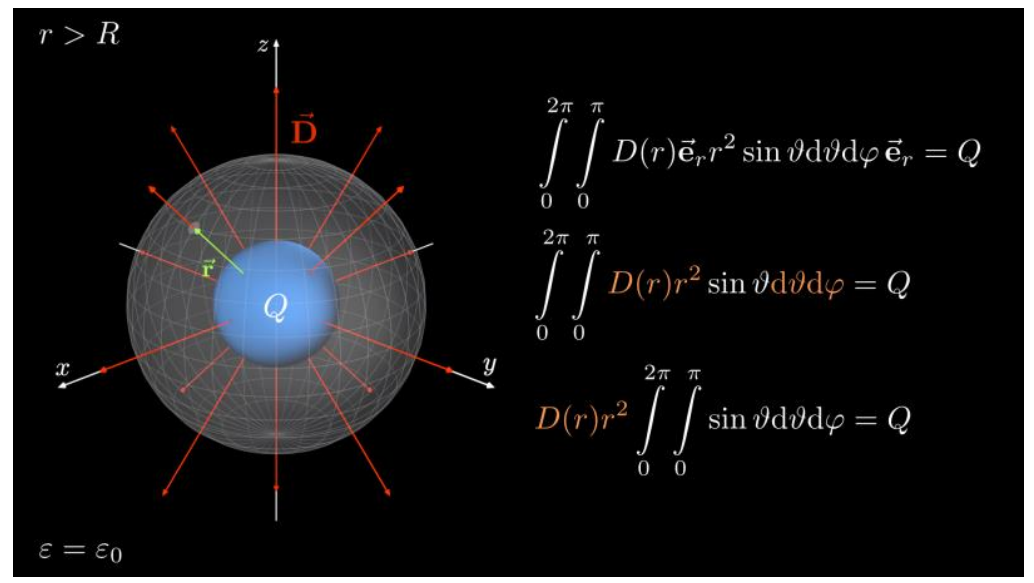

c)

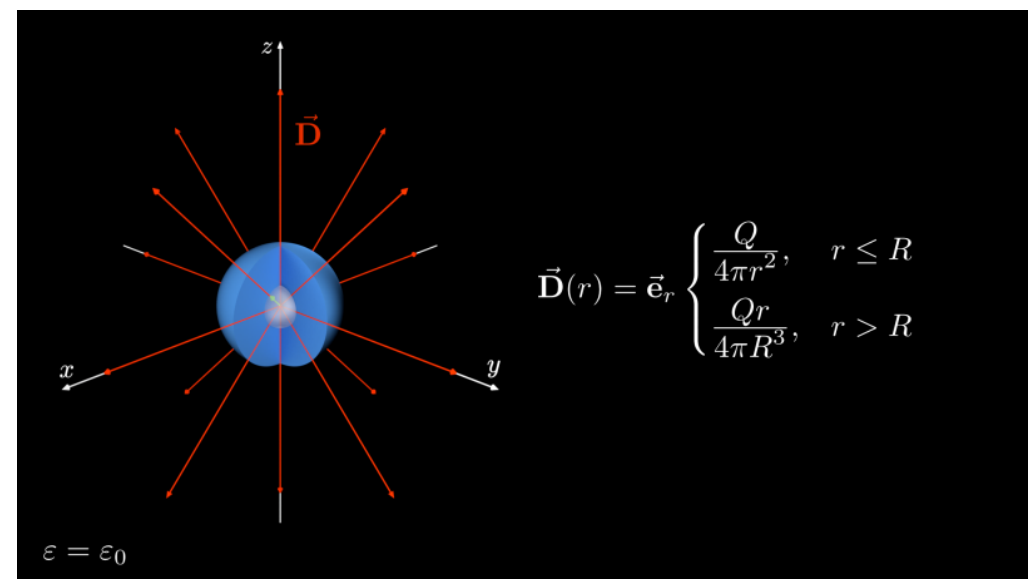

Figure 2: Selected frames from one of the teaching videos developed in this work, see text for details. 